\title{
Characterization of tea leaf metabolites dependent on tea (Camellia sinensis) plant age through ${ }^{1} \mathrm{H}$ NMR-based metabolomics
}

\author{
N. H. M. Rubel Mozumder ${ }^{1 \dagger}$, Yeong-Ran Lee ${ }^{2 \dagger}$, Kyeong Hwan Hwang ${ }^{2}$, Min-Seuk Lee ${ }^{3}$, Eun-Hee Kim ${ }^{4}$ \\ and Young-Shick Hong ${ }^{1 *}$ (D)
}

\begin{abstract}
The chemical or metabolic compositions of tea (Camellia sinensis) varies according to numerous factors, such as geographical origin, cultivar, climate, plucking position, and horticultural practices. However, how the age of tea plants affects the metabolite compositions of tea leaves has not been reported yet. Therefore, we extended the metabolomic approach to the investigation of the age-related differences of tea leaf metabolites in the fresh leaves collected from tea plants aged 8 and 25 years. Multivariate statistical analysis with comprehensive metabolite profiles analyzed by ${ }^{1} \mathrm{H}$ NMR spectroscopy showed the clear metabolic differentiation between the fresh tea leaves from different ages of the tea plants. Of the various tea leaf metabolites varied according to the age of the tea plants, theanine, glutamine, catechin, and gallocatechin were uniquely dependent on the age of tea plants, demonstrating a difference of theanine metabolism between young and old tea plants. These results suggest that leaves from 25-year-old tea plants would still be worthy as a functional ingredient for the production in the food or cosmetic industry rather than quality-enhanced tea infusions for human consumption.
\end{abstract}

Keywords: Camellia sinensis, Tea, Age, Metabolomics, NMR

\section{Introduction}

Tea is one of the most widely consumed beverages in the world. The five types of teas, such as black, oolong, green, white, and pu-erh teas, are commonly available in the market, in which tea leaves are the typical ingredients $[1,2]$. Tea has been known to have health benefits such as reduced cancer risk [3], neuroprotective effects [4], and anti-inflammatory activity with compounds rich in polyphenols, catechin and its derivatives, caffeine and theanine, and minor compounds, including amino acids, theaflavins, and thearubigins [5]. In addition to health benefits, modern cosmetic industries use tea as an

\footnotetext{
*Correspondence: chtiger@jnu.ac.kr

${ }^{\dagger}$ N. H. M. Rubel Mozumder and Yeong-Ran Lee equally contributed to this work

${ }^{1}$ Division of Food and Nutrition, Chonnam National University, Yongbong-ro, Buk-gu, Gwangju 61186, Republic of Korea Full list of author information is available at the end of the article
}

ingredient for improving the quality of cosmetics products, usually termed 'cosmeceuticals' [6].

The tea plant is a perennial and economic crop with a productive lifespan that can range up to 100 years [7]. Several studies stressed the importance of peak tea yields and their low productive-driver reasons. For example, the effects of a tea plant's genotype and environment, management practices, and the interactions between the factors [8] on productivity have been reported, as well as the effect of tea plant age [9]. Notably, the age of tea plants has a substantial effect on tea yield and peak yields, which are optimum between the ages of 20 and 40 years and then decline afterward [10]. Therefore, the age of tea plants may be a topic of immense interest for research on the chemical composition of the tea leaf.

Metabolomic approaches have been reported as useful and promising tools to examine the changes in global metabolites of tea plants and potentially provide a better 
physiological understanding of the biochemical and cellular events $[11,12]$. For instance, a recent proton nuclear magnetic resonance $\left({ }^{1} \mathrm{H}\right.$ NMR) based global metabolic profiling study with green tea demonstrated that the tea metabolome was clearly dependent on growing area and hence was influenced by climatic conditions [13]. Moreover, a direct association between tea leaf metabolites and environmental factors, such as plucking seasons and climate variables, has been characterized by a ${ }^{1} \mathrm{H}$ NMR-based metabolomics approach [14]. Furthermore, a metabolomic approach revealed the metabolic dependence of tea leaves on plucking positions [15]. Although ${ }^{1} \mathrm{H}$ NMR-based metabolomic study with different types of teas provides much useful information for producing distinct tea products, the distinctions of tea leaf metabolites according to the age of tea plants remains unknown. Therefore, in the present study, we explored the agerelated differences in tea metabolites in the fresh leaves collected from tea plants aged 8 and 25 years through ${ }^{1} \mathrm{H}$ NMR-based metabolomics approach.

\section{Materials and methods Origin of tea samples}

Tea plants (Camellia sinensis var. Yabukita) of two different ages (8- and 25-year-old) analyzed in this study were grown at the same tea garden located in Seogwang $\left(33^{\circ} 18^{\prime} 17.67^{\prime \prime} \mathrm{N}, 126^{\circ} 17^{\prime} 42.97^{\prime \prime} \mathrm{E}\right)$, Jeju-do, Republic of Korea. Fresh tea leaves were plucked at ten different parcels on May 14, 2015, and April 24, 2016, immediately kept in dry ice, sent to Chonnam National University (CNU, Republic of Korea), and stored at $-80{ }^{\circ} \mathrm{C}$ until further analysis.

\section{Extraction of tea samples}

Tea leaves were extracted according to the protocol explained in the previously published article [16]. The frozen tea leaves without stems were ground with a mortar and pestle under liquid nitrogen. The ground tea was transferred into a plastic tube with a spatula and freeze-dried at $-80{ }^{\circ} \mathrm{C}$ for $48 \mathrm{~h}$. Then freeze-dried samples of $10 \mathrm{mg}$ were dissolved in a mixture of methanol$d_{4}\left(\mathrm{CD}_{3} \mathrm{OD}, 490 \mu \mathrm{L}\right)$ and deuterium water $\left(\mathrm{D}_{2} \mathrm{O}, 210 \mu \mathrm{L}\right)$ in $1.5 \mathrm{~mL}$ Eppendorf tubes. After that, the mixture was sonicated at $25^{\circ} \mathrm{C}$ for $20 \mathrm{~min}$ followed by centrifugation at $13,000 \mathrm{rpm}$ for $15 \mathrm{~min}$ at $10^{\circ} \mathrm{C}$. The resultant supernatants were transferred to 5-mm NMR tubes.

\section{${ }^{1} \mathrm{H}$ NMR spectroscopic analysis of tea extracts}

For ${ }^{1} \mathrm{H}$ NMR spectroscopic analysis, $550 \mu \mathrm{L}$ of the supernatant was transferred into 5-mm NMR tubes, and a ${ }^{1} \mathrm{H}$ NMR spectrum was acquired on a Bruker Avance 700 spectrometer (Bruker Biospin, Rheinstetten, Germany) operating at $700.40 \mathrm{MHz}{ }^{1} \mathrm{H}$ frequency and a temperature of $298 \mathrm{~K}$, equipped with a cryogenic tripleresonance probe and a Bruker automatic injector. A one-dimensional (1D) NMR spectrum of tea leaf extract was acquired with the 1D nuclear Overhauser effect spectrometry (NOESY) pulse sequence with water presaturation. The signal assignment of the tea leaf extract was facilitated by two-dimensional (2D) total correlation spectroscopy (TOCSY), heteronuclear single quantum correlation (HSQC), spiking experiments with reference compounds, and comparisons of chemical shifts reported in previous work or a database. Furthermore, onedimensional statistical total correlation spectroscopy (STOCSY) [17] was also used for the signal assignment.

\section{NMR data processing and multivariate statistical analysis}

All ${ }^{1} \mathrm{H}$ NMR spectra derived from tea leaf extracts were corrected manually for the phase and baseline distortions using TOPSPIN software (Version 4.04, Bruker BioSpin, Rheinstetten, Germany), converted to ASCII format, and then imported into MATLAB (R2010b; The Mathworks, Inc., Natick, MA). The $i$ coshift method [18] was applied to align the 1D NMR spectra in full resolution. After the alignment of the 1D NMR spectra, regions of the NMR spectra corresponding to the solvent methanol (3.36-3.40 ppm) and residual water (4.80-4.90 ppm) were removed. Probabilistic quotient normalization of the spectra using the median spectrum was carried out after total integral normalization to avoid dilution effects of samples and effects of metabolites [19]. The resultant datasets were then imported into SIMCA-P version 14.0 (Umetrics, Umeå, Sweden) and applied to a mean centering scaling method for multivariate statistical analysis. First, the principal component analysis (PCA), an unsupervised pattern recognition method, was performed to examine intrinsic variation in the dataset. After that, a supervised pattern recognition method, orthogonal projection on latent structure-discriminant analysis (OPLSDA) [20] was used to extract maximum information on discriminant compounds from the spectra. An in-house MATLAB script developed by Imperial College London, UK, was used for pairwise comparison between two groups of classes in OPLS-DA loading plots, in which the loading plots corresponding to the correlation coefficients between variables and classes were combined by back-transformed loading together with variable weights. A color code visualized the concentration differences and discrimination weights between the classes in the OPLSDA model that corresponds to the square correlation coefficient in the OPLS-DA loadings as described by [21]. A permutation test of 200 times repetitions along with seven-fold cross-validation was performed to validate the 
OPLS-DA model. The $\mathrm{Q}^{2}$ values generated from the permutation test were compared to the $\mathrm{Q}^{2}$ values of the real model. If the maximum value of $\mathrm{Q}^{2}$ from the permutation test was smaller than the $\mathrm{Q}^{2}$ of the real model, the model was considered to be a predictable model. $\mathrm{R}^{2} \mathrm{X}$ was used to evaluate possible overfitting of the model. Hence, the quality of the models is described by $R^{2} X$ and $\mathrm{Q}^{2}$ values. $\mathrm{R}^{2} \mathrm{X}$ is defined as the proportion of variance in the data explained by the models and indicates goodness of fit, and $Q^{2}$ is defined as the proportion of variance in the data predictable by the model and indicates predictability.

\section{Statistical analysis}

Statistical analyses of the data were conducted using the software package SPSS (IBM SPSS Statistics ver. 23; SPSS Corp., USA). Significance in the relative contents of tea leaf metabolites was evaluated by one-way analysis of variance (ANOVA) followed by Duncan's multiple-range test. A probabilistic value of $P<0.05$ was considered to be statistically significant. The integral area of the peaks identified in $1 \mathrm{D}^{1} \mathrm{H}$ NMR that corresponded to non-overlapped individual metabolites was examined to quantify the relative comparisons of the levels of individual metabolites.
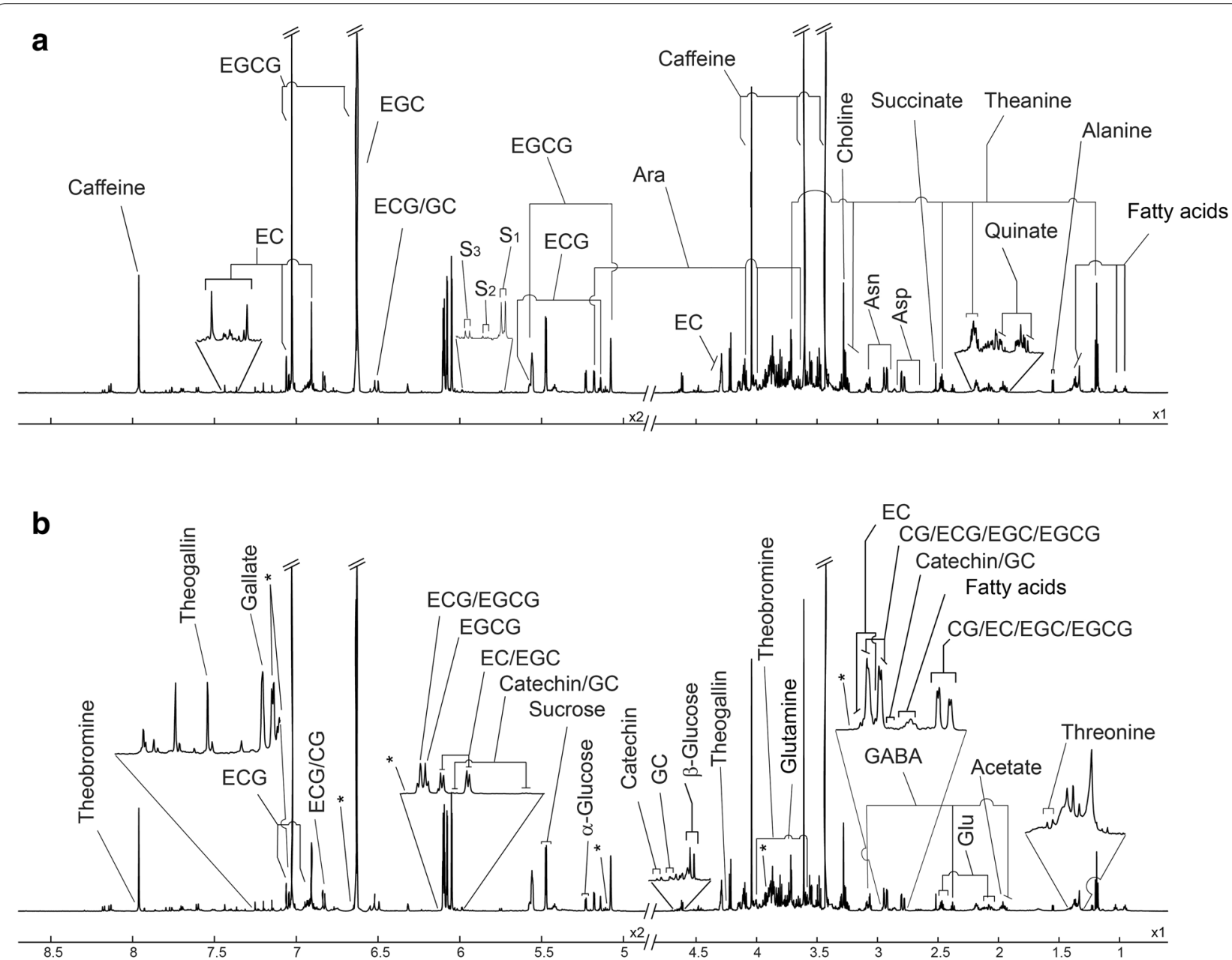

\section{Chemical shift $\left({ }^{1} \mathrm{H}, \mathrm{ppm}\right)$}

Fig. 1 Representative ${ }^{1} \mathrm{H} 700 \mathrm{MHz}$ NMR spectra of tea leaf extracts from 8-year-old (a) and 25-year-old (b) tea (C. sinensis) plants. The tea metabolites identified by ${ }^{1} \mathrm{H}$ NMR spectroscopy include acetate, alanine, 2-O-( $\beta$-L-arabinopyranosyl)-myo-inositol (Ara), asparagine (Asn), aspartate (Asp), caffeine, catechin, choline, epicatechin (EC), epicatechin gallate (ECG), epigallocatechin (EGC), epigallocatechin gallate (EGCG), fatty acids, $\gamma$-aminobutyric acid (GABA), gallate, gallocatechin (GC), a-glucose, $\beta$-glucose, glutamate (Glu), glutamine, quinate, sucrose, succinate, theanine, theobromine, theogallin, and threonine. S1, S2, and S3 were tentatively assigned as sugar compounds. Asterisks represent epigallocatechin 3-O-(3"-O-methyl) gallate (EGCG3"Me) 


\section{Results}

\section{${ }^{1} \mathrm{H}$ NMR spectroscopic analysis of tea leaves}

To understand the global metabolic dynamics in tea leaves as tea (C. sinensis) plants become aged, representative ${ }^{1} \mathrm{H}$ NMR spectra of tea leaves collected from 8 -yearold (a) and 25-year-old (b) tea plants plucked in May 2015 and April 2016, respectively, are shown in Fig. 1. The $1 \mathrm{D}{ }^{1} \mathrm{H}$ NMR spectra consisted of a diverse range of tea leaf metabolites, including acetate, alanine, $2-O-(\beta-\mathrm{L}-$ arabinopyranosyl)-myo-inositol (Ara), asparagine, aspartate, caffeine, choline, fatty acids, gallate, $\gamma$-aminobutyric acid (GABA), $\alpha$-glucose, $\beta$-glucose, glutamine, glutamate, quinate, sugars, sucrose, succinate, catechin, gallocatechin $(\mathrm{GC})$, epicatechin (EC), epicatechin gallate (ECG), epigallocatechin (EGC), epigallocatechin gallate (EGCG), epigallocatechin 3-O-(3"-O-methyl) gallate (EGCG3"Me), theanine, theobromine, theogallin, threonine, and valine. These tea leaf metabolites were assigned by spiking with the pure chemicals and also by comparing the data from the published literature $[13-15,22,23]$. The metabolites assignment was also validated by 2D TOCSY and HSQC NMR experiments, as described by previous studies [15, 22]. Additional file 1: Table S1 provides the chemical shifts of tea leaf metabolites and their corresponding multiplicity or coupling constant from tea leaves assigned by TOCSY and HSQC NMR experiments.

\section{Metabolic differentiations of tea leaves according to plant age}

A pattern recognition method by multivariate statistical analysis, such as principal component analysis (PCA) and orthogonal projection on latent structure-discriminant analysis (OPLS-DA), was employed for the entire ${ }^{1} \mathrm{H}$ NMR dataset for visualizing the global differences in tea leaf metabolites according to age of tea plant. An unsupervised PCA model was used to see the initial spectral features of the ${ }^{1} \mathrm{H}$ NMR dataset and the metabolic relationships between tea samples. The PCA model showed the metabolic dependence of tea leaves on growing vintage or year described by the first principle component with $67.3 \%$ variations and on the age of tea plants explained by the second principal component with $9.83 \%$ (Fig. 2a). These metabolic dependences were more clear in the OPLS-DA model, as shown in Fig. 2b. The tea leaves collected in 2015 and 2016 were further differentiated in the corresponding OPLS-DA models, as shown in Fig. 2c, $\mathrm{d}$, respectively, which demonstrated strong dependences of tea leaf metabolites on the age of tea plants.

\section{Identification of tea leaf metabolites associated with the age of tea plants}

A pairwise OPLS-DA model was generated with one predictive and one orthogonal component to identify the tea leaf metabolites responsible for metabolic differentiations according to the age of the tea plants (Fig. 3). Clear differentiation between the tea samples of different ages was observed in all OPLS-DA score plots with a high predictability $\left(Q^{2}\right)$ and high goodness of fit $\left(R^{2} X\right)$, which accounted for 0.60 and 0.85 between the tea leaves of the 8- and 25-year-old tea plants that were harvested in 2016 (Fig. 3a), and 0.66 and 0.93 between the tea leaves of the 8- and 25-year-old tea plants that were harvested in 2015 (Fig. 3c). All these OPLS-DA models were validated by permutation tests (Additional file 1: Fig. S2). The upper sections of the OPLS-DA loading plots represent the tea leaf metabolites that were higher in the old tea plants than in the young tea plants, whereas the lower sections were characterized by lower contents of tea leaf metabolites in the old tea plants (Fig. 3b, d). The different colors on the OPLS-DA loading plots explain the significant differences in metabolites responsible for differentiations between the young and old tea plants, and a correlation coefficient of the OPLS-DA plot greater than 0.45 was considered to be significant, as described in our previous studies [15]. The OPLS-DA loading plot with tea leaves between 8- and 25-year-old tea plants collected in 2016 showed lower levels of alanine (Ala), 2-O-( $\beta$-L-arabinopyranosyl)-myo-inositol (Ara), caffeine, epicatechin (EC), epigallocatechin (EGC), epigallocatechin 3-O-(3"-O-methyl) gallate (EGCG3"Me), leucine, $\alpha$-glucose, $\beta$-glucose, glutamine $(G \ln )$, quinate, succinate, sucrose, theanine, theobromine in tea leaves from the 25-year-old tea plants (Fig. 3b). On the other hand, the tea leaf metabolites responsible for differentiations between 8- and 25-year-old tea plants plucked in 2015 were identified in the OPLS-DA loading plot (Fig. 3d). Therefore, tea leaves collected from 25-year-old tea plants in 2015 were characterized by lower levels of acetate, catechin, GABA, gallate, theanine, glutamine, and theogallin compared to 8-year-old tea plants (Fig. 3d). Quantitative differences of individual metabolites according to the age of tea plants were calculated from a total integral area of ${ }^{1} \mathrm{H}$ NMR spectra corresponding to each metabolite (Fig. 4).

\section{Discussion}

Metabolomics has already unveiled the metabolic profiles of tea leaves (C. sinensis), which were perturbed according to several factors, including plucking positions and seasons, climates, cultivation conditions, and cultivars $[11,13,14,22,23]$. However, information about the global metabolite differences between tea leaves according to the age of tea plants is very limited. Thus, in order to characterize the age-related metabolites in tea leaves, ${ }^{1} \mathrm{H}$ NMR-based metabolomics was employed with 8- and 25-year-old tea plants collected in 2015 and 2016. 

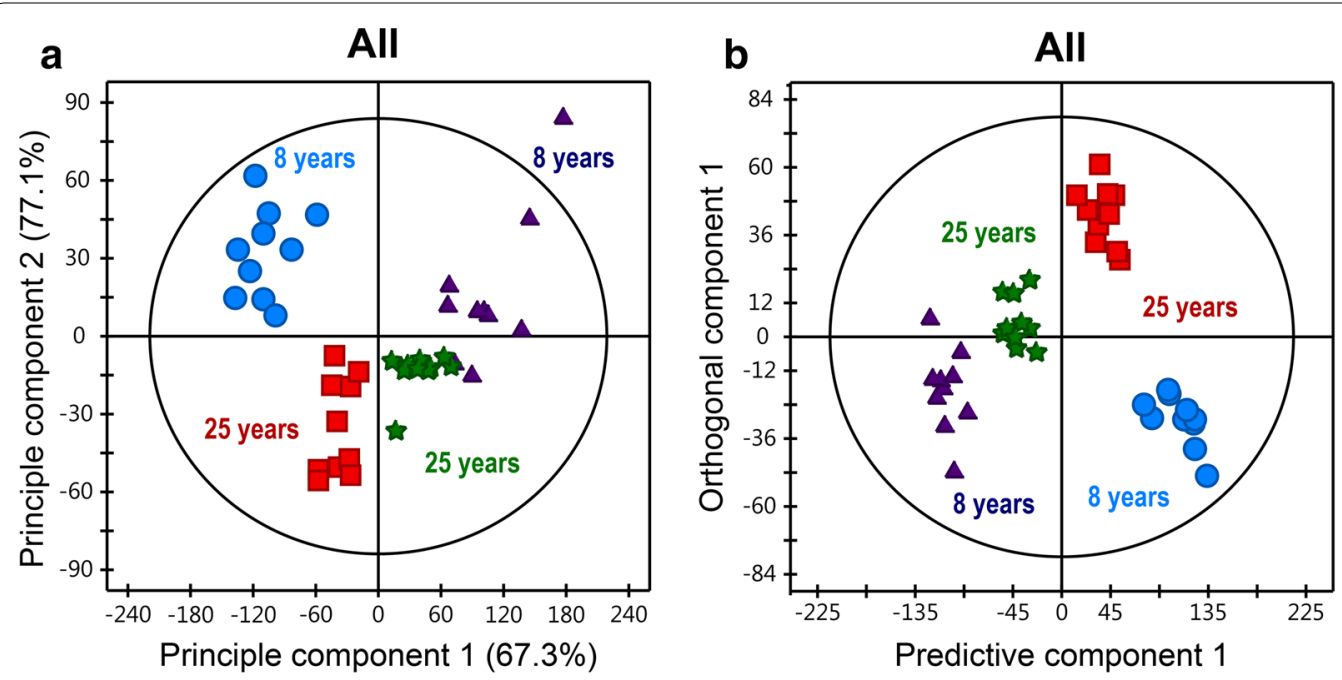

8-year-old (2015)

25-year-old (2015)

A 8-year-old (2016)

$\downarrow$ 25-year-old (2016)

C

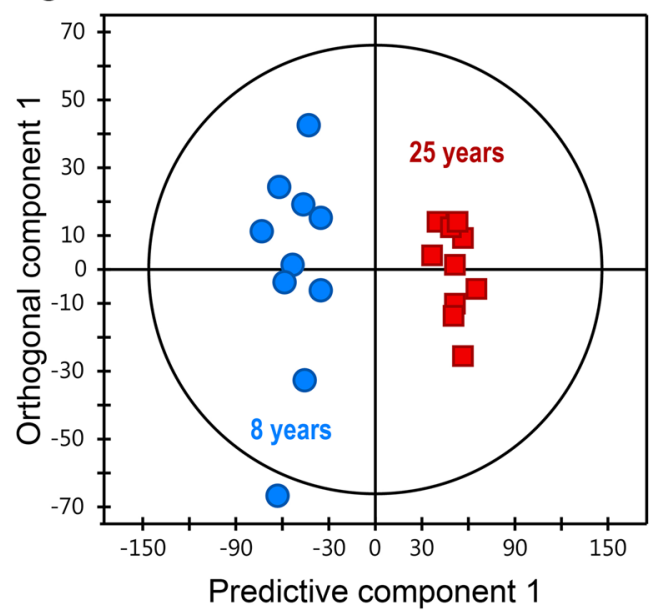

d

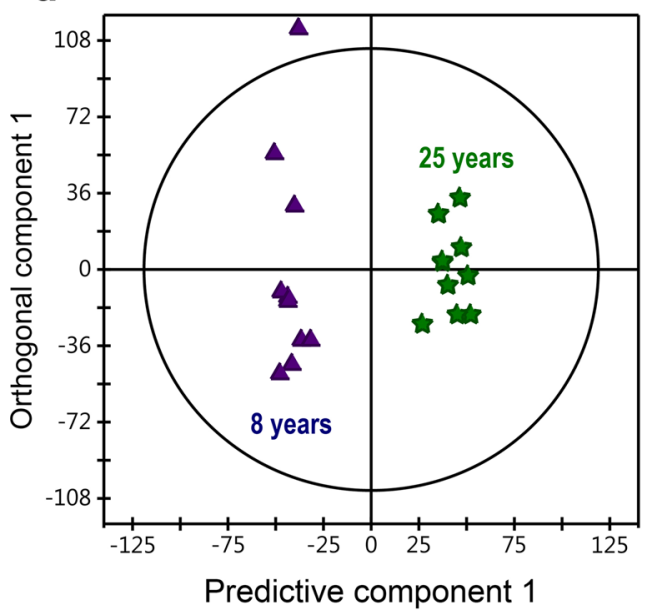

Fig. 2 PCA (a) and OPLS-DA (b-d) score plots derived from 700 MHz ${ }^{1}$ H NMR spectra of tea leaf extracts obtained in May 2015 and April 2016 , demonstrating a clear dependence of tea leaf metabolome on the age of tea plants

Tea leaf metabolites associated with the age of tea plants were clearly different between 2015 and 2016, may be due to different plucking seasons of April in 2016 and May in 2015 (Fig. 3), even though the first harvest of the tea leaves is performed with unique criteria, such as amounts of total amino acids and the number of buds every year. Therefore, we speculate that the low rate of photosynthesis and then limited carbohydrate metabolism in old tea plants during April 2016, which were indicated by reduced glucose and sucrose, might reduce the synthesis of other tea metabolites, such as alanine, succinate, glutamine, theanine, EC, EGC, catechin, caffeine, and theobromine. However, many of the tea leaf metabolites not differ between 8- and 25-year-old tea plants during May 2015, perhaps because of different climate conditions between
April 2016 and May 2015. The associations between tea leaf metabolites and climate conditions in several years have been reported in our previous study [13].

Of the various tea leaf metabolites perturbed according to the age of the tea plants, theanine, glutamine, catechin, and gallocatechin were uniquely dependent on the age of the tea plants (Fig. 4). Theanine in tea plants is produced from glutamic acid and ethylamine in shoots and converted into catechins in leaves [24, 25]. Therefore, high accumulations of theanine, glutamine, a precursor of glutamic acid, and catechin in leaves of young tea plants indicate reduced or lower metabolism of theanine in young tea plants than in old tea plants. On the other hand, these tea leaf metabolites would be highly metabolized in old tea plants, as evidenced by a high accumulation 

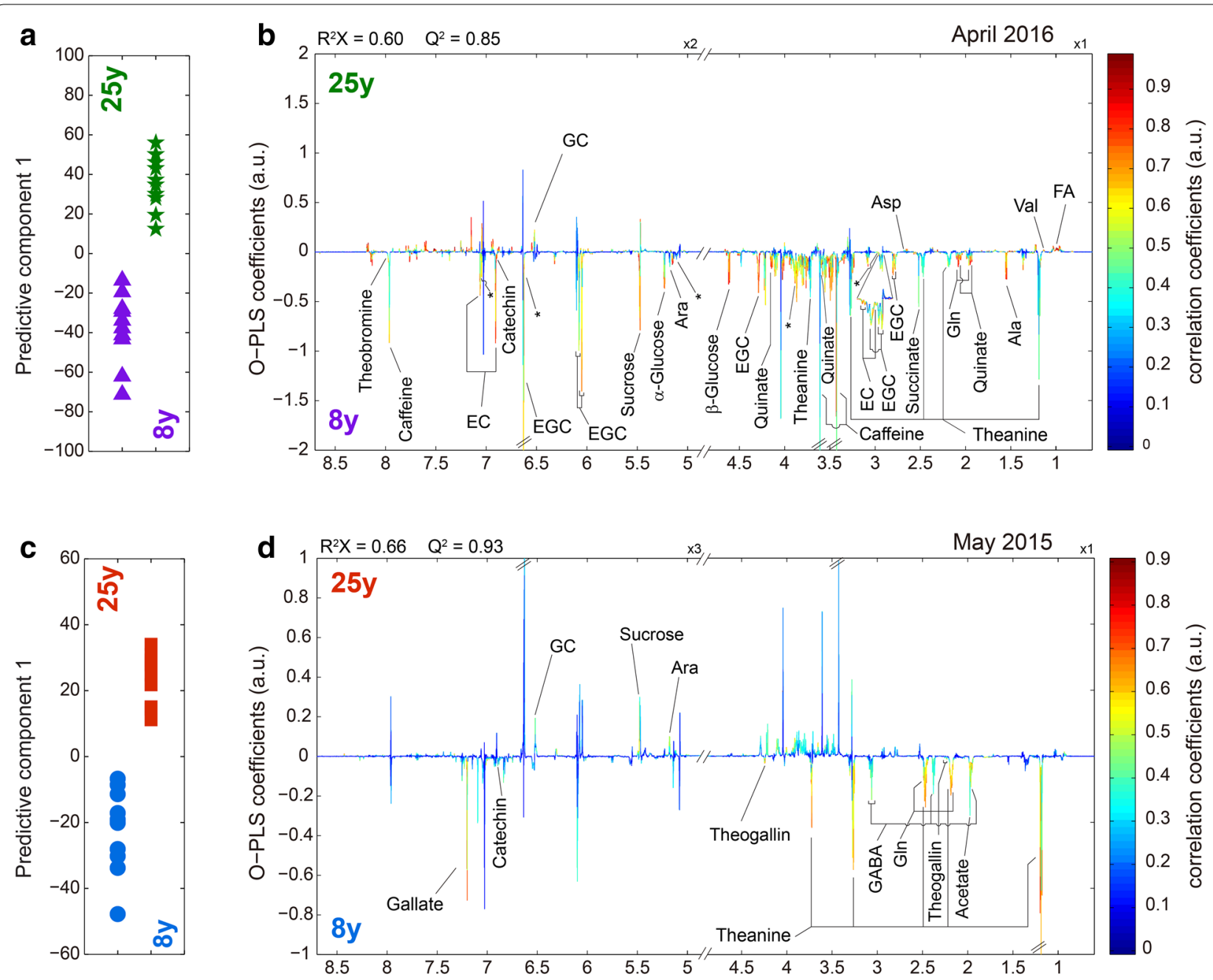

Fig. 3 The OPLS-DA score (a and $\mathbf{c}$ ) and loading $(\mathbf{b}$ and $\mathbf{d})$ plots derived from ${ }^{1} \mathrm{H}$ NMR spectra of tea leaf extracts, providing a pairwise plot for metabolic comparison between tea plants aged 8 ( 8 y) and 25 (25 y) years old collected in April 2016 (a and b) and in May 2015 (c and d). In the loading plot (b and $\mathbf{d}$ ), the upper section represents the higher levels of tea leaf metabolites in old tea plants compared to young tea plants, whereas the lower section denotes metabolites lower in old tea plants. The color code in the loading plot corresponds to the correlation between the variables. All OPLS-DA modes were generated with one predictive component and one orthogonal component and were validated by a permutation test (Additional file 1: Fig. S2). Ala, alanine; Ara, 2-O-( $\beta$-L-arabinopyranosyl)-myo-inositol; Asp, aspartic acid; EC, epicatechin; EGC, epigallocatechin; FA, fatty acids; GABA, $\gamma$-aminobutyric acid; GC, gallocatechin; Gln, glutamine; Val, valine. Asterisks represent epigallocatechin 3-O-(3"-O-methyl) gallate (EGCG3"Me)

of gallocatechin in leaves of old tea plants (Fig. 4P). The metabolite perturbations of tea leaves observed in different ages of tea plants in the current study should be distinct from those related to plucking positions because tea leaves collected according to plucking positions contain both young and old tea leaves [15]. For example, the first tea leaves of the five-tea leaf buds represent the youngest tea leaves, whereas the last tea leaves are the oldest tea leaves.

In conclusion, a unique metabolite marker in tea leaves to characterize old tea plants was found through a ${ }^{1} \mathrm{H}$ NMR-based metabolomics approach with 8and 25-year-old tea plants. The tea leaf metabolites, including theanine, glutamine, catechin and gallocatechin were strongly associated with the age of tea plants, demonstrating differences of theanine metabolism between young and old tea plants. The current study to characterize global metabolite differences of old tea plants and then assess their quality suggests that old tea plants would still be worthwhile for producing functional ingredients of various major catechin compounds, such as EC, EGCG, EGC, ECG, and EGCG3"Me, because their metabolisms were not dependent on the age of the tea plants, even though there are low amounts of theanine in their leaves. 


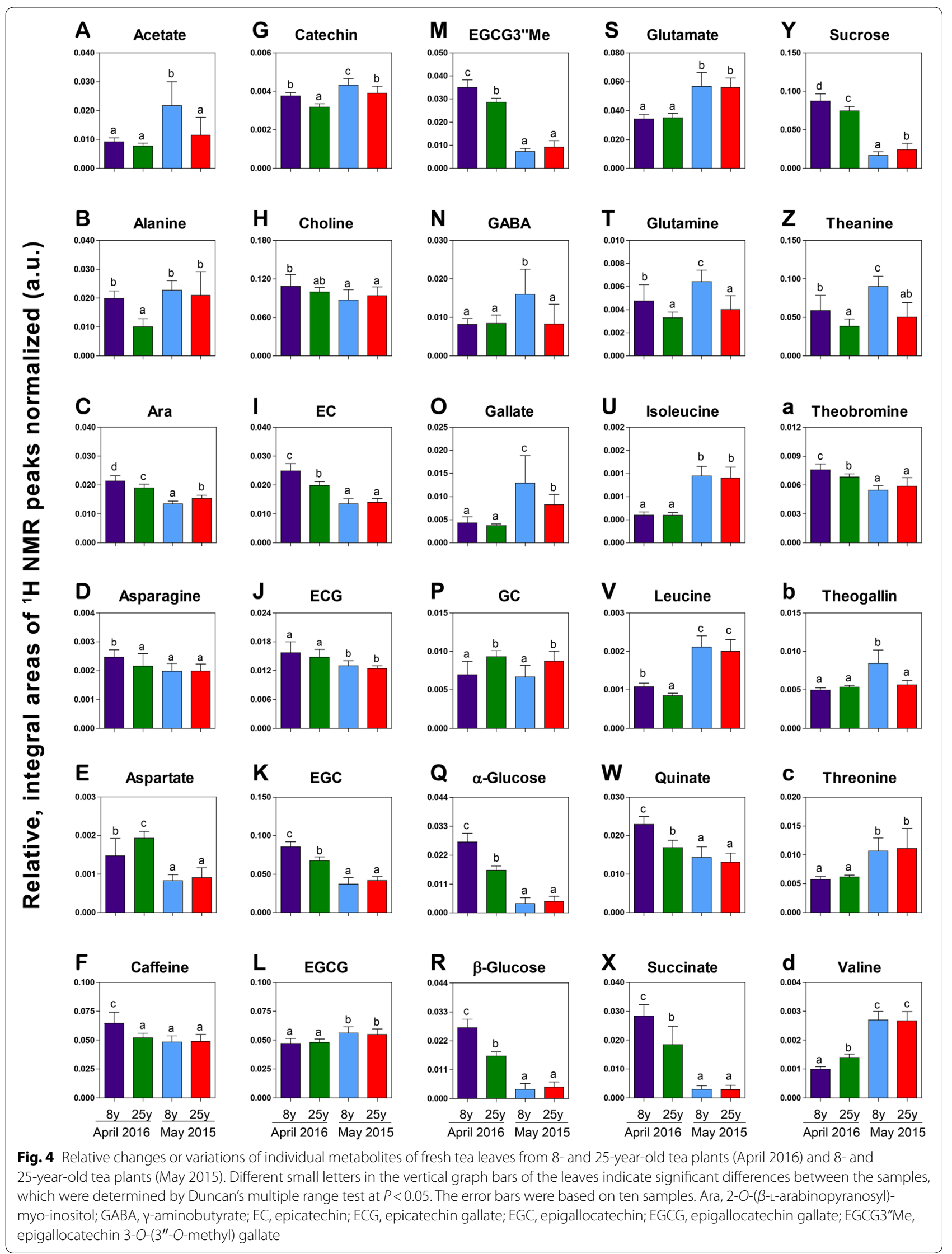




\section{Supplementary information}

Supplementary information accompanies this paper at https://doi. org/10.1186/s13765-020-0492-7.

Additional file 1:Table S1. Assignments of Metabolites in the Representative ${ }^{1} \mathrm{H}$ NMR Spectra. Fig. S1. Representative ${ }^{1} \mathrm{H} 700 \mathrm{MHz}$ NMR spectra of tea leaf extract from 8- and 25-year-old tea plants plucked on May 2015. Fig. S2. Permutation tests of PLS-DA models.

\section{Abbreviations}

$\mathrm{CD}_{3} \mathrm{OD}$ : methanol- $d_{4} ; \mathrm{D}_{2} \mathrm{O}$ : deuterium water; Asn: asparagine; Asp: aspartate; ANOVA: one-way analysis of variance; Ara: $2-O-(\beta$-L-arabinopyranosyl)-myoinositol; EC: epicatechin; ECG: epicatechin gallate; EGC: epigallocatechin; EGCG : epigallocatechin gallate; EGCG3"Me: epigallocatechin 3-O-(3"-O-methyl) gallate; FA: fatty acids; GC: gallocatechin; Glu: glutamine; ${ }^{1} \mathrm{H}$ NMR: proton nuclear magnetic resonance; HSQC: heteronuclear single quantum correlation; NOESY: nuclear Overhauser effect spectrometry; OPLS-DA: orthogonal projection on latent structure-discriminant analysis; PCA: principal component analysis; S1: sugar 1; S2: sugar 2; S3: sugar 3; STOCSY: statistical total correlation spectroscopy; TOCSY: total correlation spectroscopy; ID: one-dimensional; 2D: two-dimensional.

\section{Acknowledgements}

We would like to thank the Korea Basic Science Institute (KBSI) under the R\&D program (Project No. C030440) supervised by the Ministry of Science and ICT.

\section{Authors' contributions}

MR, YSH and YRL analyzed the data and wrote the manuscript; $\mathrm{KHH}$ and MSL collected the tea materials; EHK performed the experiments; MR and YSH revised the manuscript. All authors read and approved the final manuscript.

\section{Funding}

Not applicable.

\section{Availability of data and materials \\ Not applicable.}

\section{Competing interests}

The authors declare that they have no competing interests.

\section{Author details}

${ }^{1}$ Division of Food and Nutrition, Chonnam National University, Yongbong-ro, Buk-gu, Gwangju 61186, Republic of Korea. ${ }^{2}$ Basic Research \& Innovation Division, R\&D Center, AmorePacific Corporation, Yongin-si, Gyeonggi-do 446-729, Republic of Korea. ${ }^{3}$ Osulloc Tea R\&D Center, Osulloc Farm Corporation, Jeju 699-820, Republic of Korea. ${ }^{4}$ Center for Research Equipment, Korea Basic Science Institute, Cheongwon-Gu, Cheongju-Si, Chungbuk 363-883, Republic of Korea.

Received: 2 November 2019 Accepted: 17 January 2020

Published online: 06 February 2020

\section{References}

1. Graham HN (1992) Green tea composition, consumption, and polyphenol chemistry. Prev Med 21(3):334-350

2. Harbowy ME, Balentine DA (1997) Tea chemistry. Crit Rev Plant Sci 16(5):415-480

3. Kuroda Y, Hara Y (1999) Antimutagenic and anticarcinogenic activity of tea polyphenols. Mutat Res Rev Mutat 436(1):69-97

4. Almajano MP, Vila I, Gines S (2011) Neuroprotective effects of white tea against oxidative stress-induced toxicity in striatal cells. Neurotox Res 20(4):372-378

5. de Mejia EG, Ramirez-Mares MV, Puangpraphant S (2009) Bioactive components of tea: cancer, inflammation and behavior. Brain Behav Immun 23(6):721-731
6. Quay ER, Chang YC, Graber E (2017) Evidence for anti-aging South Korean cosmeceuticals. J Drugs Dermatol 16(4):358-363

7. De Costa W, Mohotti AJ, Wijeratne MA (2007) Ecophysiology of tea. Braz J Plant Physiol 19(4):299-332

8. Spiertz JHJ, Struik PC, Van Laar H (2007) Scale and complexity in plant systems research: gene-plant-crop relations, Wageningen UR Frontis series, vol 21. Springer, Berlin

9. Iori P, Da Silva RB, Ajayi AE, De Silva FA, De Junior M, Silva M, De Souza ZM (2014) What drives decline productivity in ageing tea plantation-soil physical properties or soil nutrient status. Agric Sci 2(1):22-36

10. Kamau DM, Spiertz JH, Oenema O, Owuor PO (2008) Productivity and nitrogen use of tea plantations in relation to age and genotype. Field Crops Res 108(1):60-70

11. Ji HG, Lee YR, Lee MS, Hwang KH, Park CY, Kim EH, Park JS, Hong YS (2018) Diverse metabolite variations in tea (Camellia sinensis L.) leaves grown under various shade conditions revisited: a metabolomics study. J Agric Food Chem 66(8):1889-1897

12. Tarachiwin L, Ute K, Kobayashi A, Fukusaki E (2007) ${ }^{1} \mathrm{H}$ NMR based metabolic profiling in the evaluation of Japanese green tea quality. J Agric Food Chem 55(23):9330-9336

13. Lee JE, Lee BJ, Chung JO, Kim HN, Kim EH, Jung S, Lee SJ, Hong YS (2015) Metabolomic unveiling of a diverse range of green tea (Camellia sinensis) metabolites dependent on geography. Food Chem 174:452-459

14. Lee JE, Lee BJ, Chung JO, Hwang JA, Lee SJ, Lee CH, Hong YS (2010) Geographical and climatic dependencies of green tea (Camellia sinensis) metabolites: a ${ }^{1} \mathrm{H}$ NMR-based metabolomics study. J Agric Food Chem 58(19):10582-10589

15. Lee JE, Lee BJ, Hwang JA, Ko KS, Chung JO, Kim EH, Lee SJ, Hong YS (2011) Metabolic dependence of green tea on plucking positions revisited: a metabolomic study. J Agric Food Chem 59(19):10579-10585

16. Kim HK, Choi YH, Verpoorte R (2010) NMR-based metabolomic analysis of plants. Nat Protoc 5(3):536-549

17. Cloarec O, Dumas ME, Craig A, Barton RH, Trygg J, Hudson J, Blancher C, Gauguler D, Lindon JC, Nicholson J (2005) Statistical total correlation spectroscopy: an exploratory approach for latent biomarker identification from metabolic ${ }^{1}$ H NMR data sets. Anal Chem 77(5):1282-1289

18. Savorani F, Tomasi G, Engelsen SB (2010)/coshift: a versatile tool for the rapid alignment of 1D NMR spectra. J Magn Reson 202(2):190-202

19. Dieterle F, Ross A, Schlotterbeck G, Senn H (2006) Probabilistic quotient normalization as robust method to account for dilution of complex biological mixtures. Application in $1 \mathrm{H}$ NMR metabonomics. Anal Chem 78(13):4281-4290

20. Bylesjö M, Rantalainen M, Cloarec O, Nicholson JK, Holmes E, Trygg J (2006) OPLS discriminant analysis: combining the strengths of PLS-DA and SIMCA classification. J Chemom 20(8-10):341-351

21. Cloarec O, Dumas ME, Trygg J, Craig A, Barton RH, Lindon JC, Nicholson JK, Holmes E (2005) Evaluation of the orthogonal projection on latent structure model limitations caused by chemical shift variability and improved visualization of biomarker changes in ${ }^{1} \mathrm{H}$ NMR spectroscopic metabonomic studies. Anal Chem 77(2):517-526

22. Ji HG, Lee YR, Lee MS, Hwang KH, Kim EH, Park JS, Hong YS (2017) Metabolic phenotyping of various tea (Camellia sinensis L.) cultivars and understanding of their intrinsic metabolism. Food chem 233:321-330

23. Lee JE, Lee BJ, Chung JO, Shin HJ, Lee SJ, Lee CH, Hong YS (2011) ${ }^{1}$ H NMRbased metabolomic characterization during green tea (Camellia sinensis) fermentation. Food Res Int 44(2):597-604

24. Ashihara H (2015) Occurrence, biosynthesis and metabolism of theanine ( $\gamma$-glutamyl-L-ethylamide) in plants: a comprehensive review. Nat Prod Commun 10(5):803-810

25. Zhang Q, Liu M, Ruan J (2017) Integrated transcriptome and metabolic analyses reveals novel insights into free amino acid metabolism in Huangjinya tea cultivar. Front Plant Sci 8(291):291

\section{Publisher's Note}

Springer Nature remains neutral with regard to jurisdictional claims in published maps and institutional affiliations. 